\title{
The Supercapacitor Degradation State Diagnostic System Based on LabVIEW
}

\author{
http://dx.doi.org/10.3991/ijoe.v9i3.2630 \\ Meijuan Hao, Lifeng Wu, Yong Guan, Wei Pan, Wubing Tang, Xiaojuan Li \\ Capital Normal University, Beijing, China
}

\begin{abstract}
In this thesis, we design and implement the degradation state diagnostic system for supercapacitors. In order to detect the degradation degree of supercapacitors, it is necessary to measure the changes of parameters that include capacitance, ESR (equivalent series resistance) and the leakage current. The way to obtain these parameters is to collect the voltage and current values by the data acquisition card. And then the data are transmitted to the software of LabVIEW to calculate the parameters. By comparing the values of capacitance and ESR with the original values separately, the degradation state of the supercapacitor can be estimated.
\end{abstract}

Index Terms-supercapacitor degradation state, capacitance, ESR, leakage current

\section{INTRODUCTION}

Currently, the virtual instrument is the hotspot technology in the field of measurement and control. It represents the future development direction of the instrument technology. LabVIEW is a widely used software platform. In terms of software, it has perfect software features and a large number of test-related functions. In terms of hardware, it includes variety of signal acquisition and processing boards which can be used to meet requirements of measurement and control systems.

The electrochemical capacitor, called supercapacitor for short, is a new energy storage device between traditional capacitors and storage batteries. Compared with traditional capacitors, it has the characteristics of large capacity, high energy, and wide operable temperature range. Compared with storage batteries, it has a high ratio of power, can be charged and discharged within a short time and moreover it is environment-friendly [1-2]. With so many advantages, the supercapacitor contains huge value and potential market in the application of cars (especially electric vehicles, hybrid cars and special load vehicles), electricity, railways, communications, national defense, electronic products and so on [3]. So it draws attention of many countries. With the widespread application of supercapacitors, detecting the degradation state of the supercapacitor is also becoming increasingly important. By measuring the voltage and current data in the charging and discharging processes, it is able to get values of capacitance, ESR and the leakage current. According to the capacitance and ESR changes, we can diagnose the degradation state of the supercapacitor. So we design the system to realize the function. In the system, the PCI9812 data acquisition card is used for multi-channel data acquisition. Data processing and computing are conducted in LabVIEW.

\section{SUPERCAPACITOR}

We detect the degradation state of the supercapacitor. The classical equivalent model of the supercapacitor is shown in Figure 1. In the model, $\mathrm{C}$ is an ideal component, which represents the storage capacity of the supercapacitor; $\mathrm{R}_{\mathrm{es}}$ is ESR, which represents the internal heat loss of the supercapacitor; $R_{e p}$ is the equivalent parallel resistance, which impacts the long-term energy storage of the supercapacitor and is usually represented by the leakage current $[1,4]$. It has been found that the capacitance and ESR are key parameters which affect life and degradation state of the supercapacitor. The leakage current has little effect on the degree of the supercapacitor degradation state [5]. So we choose capacitance and ESR to evaluate the degradation state of the supercapacitor.

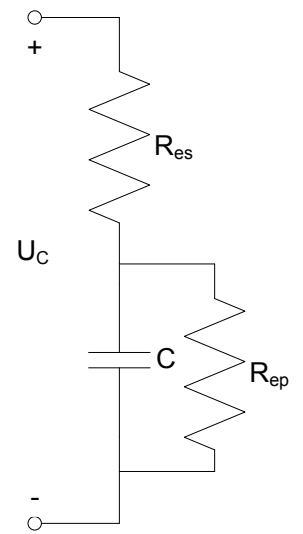

Figure 1. The classical equivalent model of the supercapacitor

There are three methods to measure the supercapacitor capacitance: constant time method, constant current charging method and constant current discharging method. We chose the second method in the system. Capacitance of capacitors follows the calculation formula.

$$
C=Q / V_{C}
$$

When the charging current of the capacitor is constant, the formula can be converted to the form as follows [6].

$$
C=I t / V_{C}
$$

It also can be expressed as follows.

$$
C=I \Delta t / \Delta V_{C}
$$


ESR has a significant influence on the efficiency and reliability of the supercapacitor. The method of constant current charging is used in the system. The voltage across the supercapacitor rises linearly during the charging process. Due to the existence of the ESR, the voltage will increase by a variable of $\mathrm{U}_{0}$ at the moment of charging. The variable of $\mathrm{U}_{0}$ is the voltage drop across the ESR [7].

$$
E S R=U_{0} / I
$$

The leakage current is generated by electric double layer ions in the charge-discharge process, which suffers the resultant force action of two forces. One is the electrostatic attraction of opposite charges in the electrode. The other is the electrolyte ion concentration gradient of the body caused by migration [8]. Leakage current always exists during the charging process. If the time is long enough, the charging current decreases to a certain value. At this moment, the charging current only compensates for the leakage current. Therefore the value of the charging current is the value of the leakage current.

When the capacitance decreases by $20 \%$, it is defined that the supercapacitor is failed. For ESR, it is twice of the original value [9-10]. When one of the two parameters, capacitance or ESR, meets the failure condition, it is defined that the supercapacitor reaches the failure state.

\section{THE OVERALL STRUCTURE OF THE SYSTEM}

The system consists of three parts which are signal conditioning circuits, the data acquisition card and the PC. Signal conditioning circuits are mainly to adjust the range of the voltage and convert the current value into a voltage value. The data acquisition card transmits the two signals to a PC. In the software, the voltage and current data are processed to get capacitance, ESR and leakage current of the supercapacitor. Thus we can detect the degradation state of the supercapacitor. The overall structure of the system is shown in Figure 2.

\section{A. Hardware design of the system}

The hardware design of the system includes the selection of the data acquisition card and signal conditioning circuits design. Data acquisition card connects to signal conditioning circuits through the probe and to a PC through the PCI bus.

\section{B. Data acquisition card}

There are two signals that need to be collected. We select the PCI9812 data acquisition card to carry out the feature of multi-channel data acquisition. The PCI9812 data acquisition card is designed with the following features. It has four single-ended analog input channels, four 12-bit A / D synchronous sampling conversion, on-board $32 \mathrm{~K}$ sampling A / D FIFO and uses bus control DMA data transmission. Its sampling rate is up to $20 \mathrm{MHz}$, thus making it able to complete high-speed data acquisition. It has two signal input ranges, $-1 \mathrm{~V} \sim+1 \mathrm{~V}$ and $-5 \mathrm{~V} \sim+5 \mathrm{~V}$. The corresponding precisions are $0.008 \mathrm{~V}$ and $0.04 \mathrm{~V}$. The voltage and current signals are necessary to be measured to get he capacitance, ESR and leakage current. The restriction is the accuracy of the ESR. The value of the ESR is several $\mathrm{m} \Omega$. The voltage of ESR is several $\mathrm{mV}$ at most. So the data acquisition card select the range of $-1 \mathrm{~V}$ $\sim+1 \mathrm{~V}$.

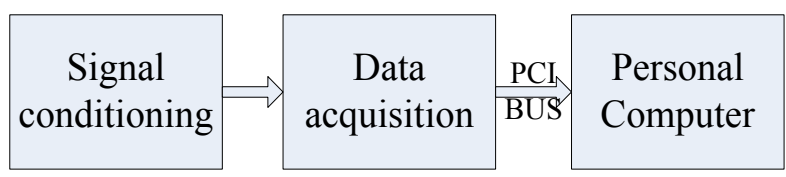

Figure 2. The overall structure of the system

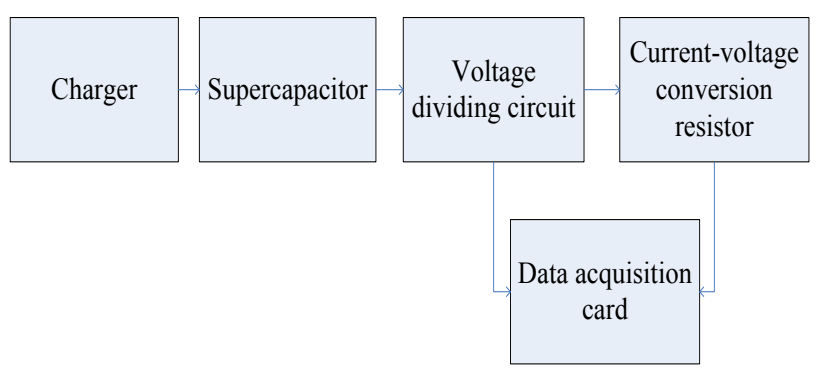

Figure 3. Signal conditioning circuits

\section{Signal conditioning circuits}

Signal conditioning circuits adjust voltage across the supercapacitor to $-1 \mathrm{~V} \sim+1 \mathrm{~V}$, and convert the charging current value to a voltage value. In this way, we can acquire the voltage data directly. The charging voltage of the supercapacitor is $2.7 \mathrm{~V}$. In the signal conditioning circuits, the voltage across the supercapacitor is decreased to $1 / 3$ of the original value. The charging current is $1 \mathrm{~A}$. A resistor with its value being $1 \Omega$, the supercapacitor and the voltage dividing circuits are connected in series. The current value is converted to a voltage value through the resistor. Thus the measured voltage value is the current value. Signal conditioning circuits is shown in Figure 3.

\section{SOFTWARE DESIGN}

The system is based on LabVIEW8.6 software platform. We use dynamic link library files compiled by VC to drive the data acquisition card. The PCI9812 data acquisition card for multi-channel data acquisition is controlled by software. The acquired data are sent to LabVIEW to calculate. After filtering, on one hand, the voltage and current value are displayed in real time. On the other hand, the data are processed and calculated to obtain the capacitance, ESR and leakage current value. By comparing the capacitance and the leakage current value with the original value separately, we can obtain the degradation state of the supercapacitor.

\section{A. Data acquisition}

The producer / consumer model is applied in the system to implement data acquisition and processing asynchronously. The data acquisition is completed in the producer loop.

The structure of the producer / consumer utilizes the data storage by means of the queue. The queue opens up a buffer, based on the first-in and first-out (First Input First Output, FIFO) principle [11]. The producer / consumer structure creates queues which can store data and extract data in a parallel manner. In this way, it is possible to avoid data losses caused by time delay. In the system, it involves both voltage and current data acquisition and processing, so we create two queues. In the queues, the storage units are arrays. 


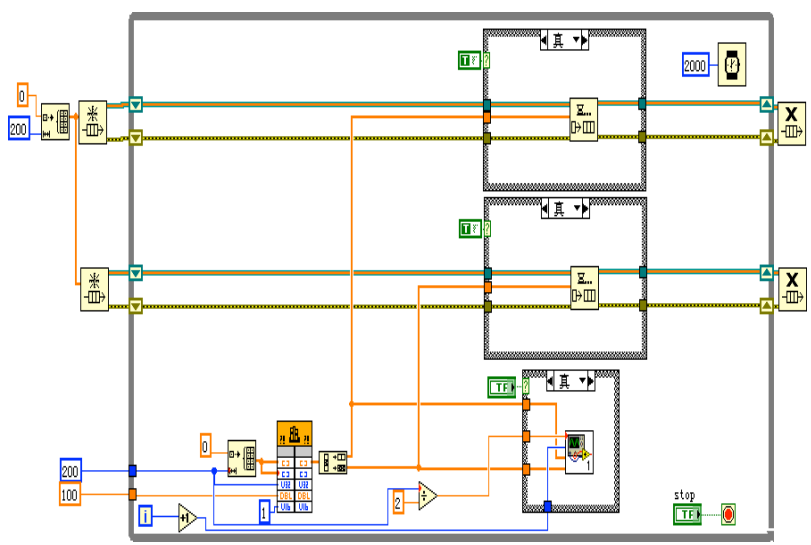

Figure 4. The specific data acquisition program

In LabVIEW, there are three methods to drive data acquisition cards: directly using LabVIEW In Port, Out Port icons; using the LabVIEW CIN icon to generate sub VI of A / D cards drivers; using LabVIEW Call Library Function icon to call dynamic link library functions of data acquisition cards [12]. Here we drive the data acquisition card by the third method. The multi-channel data acquisition program for PCI9812 data acquisition card is generated to DLL library functions for LabVIEW. The specific data acquisition program is shown in Figure 4.

\section{B. Data processing}

The data processing is completed in the consumer loop. In the producer loop, current and voltage data are splitted into two queues. The data dequeue in the consumer cycle and then are filtered. Through that we obtain capacitance, ESR and the leakage current value. Changes of the previous two parameters determine the degradation state of the supercapacitor. At the same time, the voltage and current data are displayed in real-time.

At first, filter is conducted. According to the phenomenon that the voltage and current changes in the charging process are relatively flat, and the existence of ripple and sharp pulse, Butterworth low-pass filter is selected. The passband of Butterworth low-pass filter is very smooth, and the transition zone is relatively wide. The parameters of Butterworth low-pass filter are that the order is 5 which can reduce the transition zone width ffectively and the low-pass cut-off frequency is $100 \mathrm{~Hz}$.

Then we get three parameters of the supercapacitor: capacitance, ESR and the leakage current. The flow charts are shown in Figure 5. For current data, we first remove the front data that introduced by the filter. And then we obtain the average of the rest. Next we take the data within a certain range to re-compute the average current value. The mean value of the current data is the leakage current after charging for $72 \mathrm{~h}$. In the system, we define that the current value is the leakage current when the voltage of the supercapacitor reaches $2.65 \mathrm{~V}$. And we display the leakage current. The current value is also used as the parameter in the calculation of capacitance and ESR.

In accordance with the front-end signal conditioning circuits, the voltage data are reverted. And then the same operation as the current data is performed with the voltage data. Thus we can obtain the mean value of the voltage data. We caculate the voltage values in the constant-current range, fit a straight line.The vertical axis

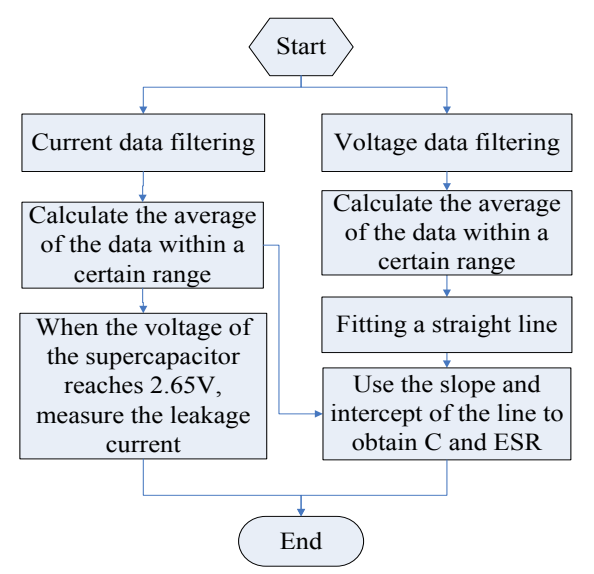

Figure 5. The flow charts

intercept of the line is the voltage drop of the ESR, the slope of the line can be expressed as the formula.

$$
S=\Delta V_{C} / \Delta t
$$

According to the formula of the capacitance, we can obtain the capacitance of the supercapacitor.

The degradation state is determined by the detected values and original values of the supercapacitor. After getting the two parameters, capacitance and ESR, we can begin to detect the degradation state of the supercapacitor. The definition of degradation value is according to the following formula.

$$
\begin{gathered}
\left(C_{0}-C_{1}\right) /\left(C_{0} \cdot 0.2\right) \cdot 100 \% \\
\left(E S R_{1}-E S R_{0}\right) /\left(E S R_{0} \cdot 2\right) \cdot 100 \%
\end{gathered}
$$

Where $\mathrm{C}_{0}$ and $\mathrm{ESR}_{0}$ represent the original values, $\mathrm{C}_{I}$ and $\mathrm{ESR}_{l}$ represent the detected values. We compare the degradation values of capacitance and ESR, and select the larger one as the degradation value. If the degradation value is 100 , the supercapacitor is failed.

\section{THE SUPERCAPACITOR TEST}

The test samples whose rated voltage is $2.7 \mathrm{~V}$ are produced by Maxwell. The factory parameters of the supercapacitor are as follows: the capacitance, ESR and leakage current are $350 \mathrm{~F}, 3.2 \mathrm{~m} \Omega$ and $0.3 \mathrm{~mA}$ separately. The voltage and current change of the supercapacitor during the charging process is shown in Figure 6. The limit value of the current is $1 \mathrm{~A}$ in the charging process. In the actual process, the charging current starts from about $0.96 \mathrm{~A}$. When the current is less than $0.95 \mathrm{~A}$, it decreases rapidly. So we define it as the constant current process when the current is greater than $0.95 \mathrm{~A}$. In the constant current charging, the voltage changes linearly.

The leakage current should be measured after charging for 72 hours. While in the experiment, it is not easy to achieve. In order to get the relationship between leakage current and time, we do the research that the leakage current changes over time with the new supercapacitor. According to the actual situation, the supercapacitor charges for 14 hours. With these data, a curve of the leakage current versus time is obtained. We select the Curve Fitting 
PAPER

The Supercapacitor Degradation State Diagnostic System Based on LabViEW

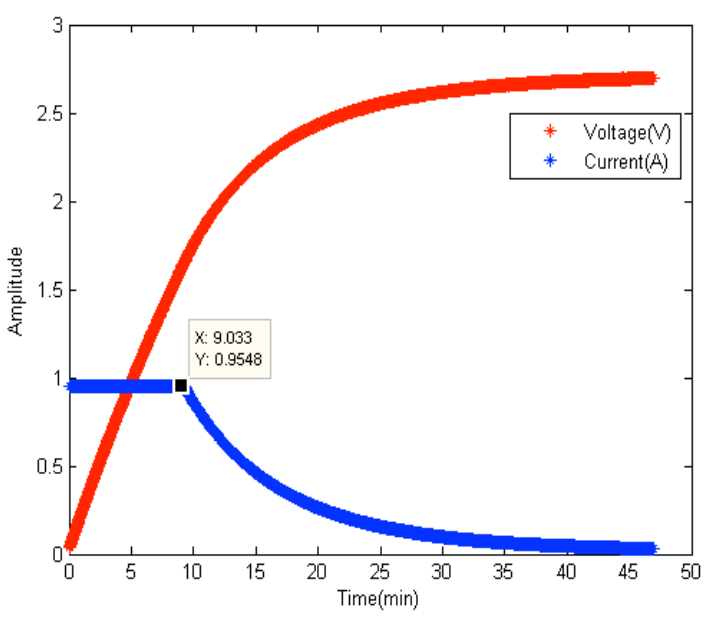

Figure 6. The charging process of the supercapacitor

Toolbox CFtool of Matlab to curve fitting. The fitting results are shown in Figure 7. The experimental results show that the charging process is completed in 45 minutes. We select the leakage current value of charging for 45 minutes to obtain the corresponding leakage current of 72 hours after the test.

\section{SYSTEM TEST}

The system test includes two parts. The first part is to detect the accuracy of the three parameters with unused supercapacitors. The second part is to detect the degradation state of the supercapacitor which has used for a period of time.

Firstly, we detect the initial state of the supercapacitor and get the parameters detection results after charging for 45 minutes. According to the regulations, the supercapacitor is normal if the capacitance does not exceed $20 \%$ of the standard value. In the detection, capacitance and ESR are $355 \mathrm{~F}, 0.0035 \Omega$ respectively. By comparing capacitance and ESR with the given supercapacitor standard values, we can see that the detection result of the capacitance and ESR value is correct.

Due to the presence of incomplete discharging phenomenon, we take the initial voltage of the supercapacitor into account. We do the test with the supercapacitor which has used for a period of time. From the experimental results, we can see that the voltage of the supercapacitor is less than $2.65 \mathrm{~V}$ after charging for $45 \mathrm{~min}$. At this moment, the leakage current has not been detected. So we choose the time when the voltage meets $2.65 \mathrm{~V}$, the test results are shown in Figure 8. As we can see from the results, ESR is significantly increasing and the constant current charging time is shortened, with using the supercapacitor.

\section{CONCLUSIONS}

The system achieves the detection of the degradation state of the supercapacitor. Based on LabVIEW, we obtain the capacitance, ESR and the leakage current in the system. And we diagnose the degradation state of the supercapacitor through the change of the capacitance and ESR. We design, test and implement the system with LabVIEW to improve the efficiency of programming compared with other programming language. The system needs to be perfected on some aspects. First, the accuracy of detecting the parameters of the supercapacitor needs to

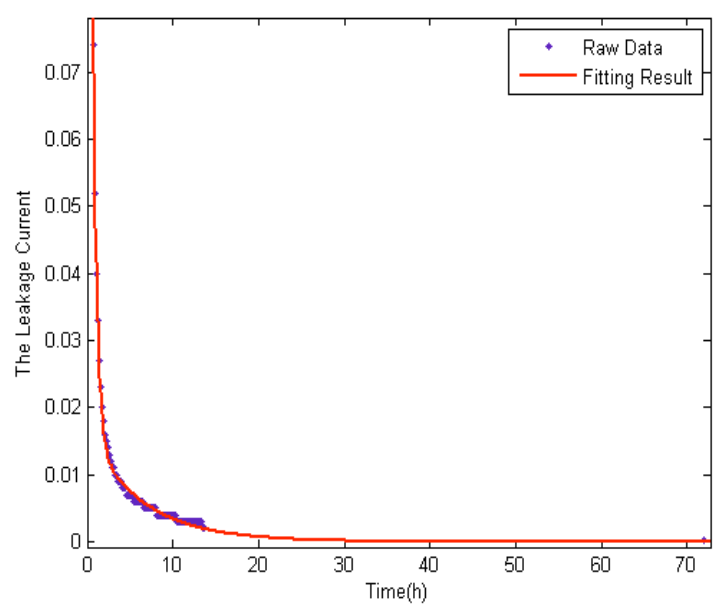

Figure 7. The leakage current versus time curving fitting

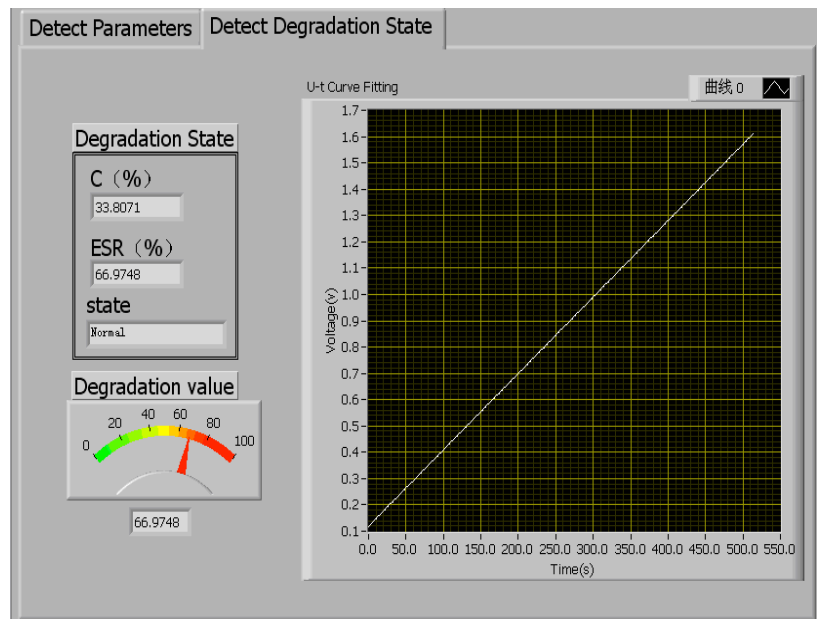

Figure 8. The test results

be improved. Next, whether the initial voltage of the supercapacitor affects the value of the parameters needs to be confirmed. We will try to solve these problems in the near future.

\section{REFERENCES}

[1] Kang Shuting, "Research of Super-capacior Test System”, Beijing Jiaotong University, 6, 2012

[2] Chen Guangzan, "Based on MCU-controlled constant-current test system", Dalian University of Technology, 6, 2009

[3] zhai Nansong, "Zhang Donglai, Research and status of supercapacitor", China Instrument Society Youth Conference Proceedings of the Ninth, China, 2007

[4] Donghwa Shin, Younghyun Kim, "Constant-current regulator-based battery-supercapacitor hybrid architecture for high-rate pulsed load applications", Journal of Power Sources, vol. 205, pp. 516-524, 2012, http://dx.doi.org/10.1016/j.jpowsour.2011.12.043

[5] R. Kötz*, P.W. Rush, "Aging and failure mode of electrochemical double layer capacitors during accelerated constant load tests", Journal of Power Sources, vol. 195, pp. 923-928, 2010 http://dx.doi.org/10.1016/j.jpowsour.2009.08.045

[6] Wang Guoqiang, Lin Zhongfu, "The main technical parameters of the testing of large capacity electric double layer capacitor", Electronic Components and Materials, vol. 19, no. 2, pp. 15-21, 2000

[7] Yao Yuying, Zhang Donglai, “A Study of ESR Testing Method for Supercapacitor", Measurement and Control Technology, vol. 24 , no. 2 , pp. $15-17,2005$

[8] Liu Zhixiang, Dong Guojun, "Perparation and performance of the electric double layer capacitor", Power Technology, vol. 25 ,no. 6, pp. $413-415,2001$ 
PAPER

The Supercapacitor Degradation State Diagnostic System Based on LABVIEW

[9] Jiang Hai, "The Study and Design of Vrla Battery Intelligent On-Line Monitoring System", Harbin University of Science and Technology, 3, 2007

[10] Amrane Oukaour, Boubekeur Tala-Ighil, "Calendar ageing and health diagnosis of supercapacitor", Electric Power Systems Research, vol. 95, pp. 330-330, 2013 http://dx.doi.org/10.1016/j.epsr. 2012.09.005

[11] Rick Bitter, Taqi Mohiuddin, "LabVIEW Advanced Programming Techniques (Second Edition) “, BocaRaton”: CRC Press LLC, 2007

[12] Xiong Huanting, "Three methods for driving data acquisition card in LabVIEW", Electrical Measurement and Instrumentation, vol. 38 , no. 8 , pp. $35-37,2001$

\section{AUTHORS}

Meijuan Hao is a graduate student of Capital Normal University in China. Her interests are the supercapacitor degradation and virtual instruments (e-mail: haomeijuan163@yeah.net).
Lifeng Wu works in highly reliable embedded system lab of Capital Normal University. Aside from MEMS sensors, control, and information fusion research, he focuses on the development of health prediction of power (e-mail: 6638752@163.com).

All authors are affiliated to College of Information Engineering, the Beijing Engineering Research Center of High Reliable Embedded System, and the Beijing Key Laboratory of Electronic System Reliability Technology at Capital Normal University, Beijing, 100048, China.

This work was supported by the National Natural Science Foundation of China (No.61070049, No.61202027), the National Key Technology R\&D Project (No. 2012DFA11340), the Beijing Natural Science Foundation of China (No.4122015), Beijing City Board of Education Science and Technology Development Project (No. KM201210028001) and Ladder Program of Beijing Key Laboratory of Electronic System Reliability Technology. Received 26 March 2013. Published as resubmitted by the authors 12 June 2013 . 